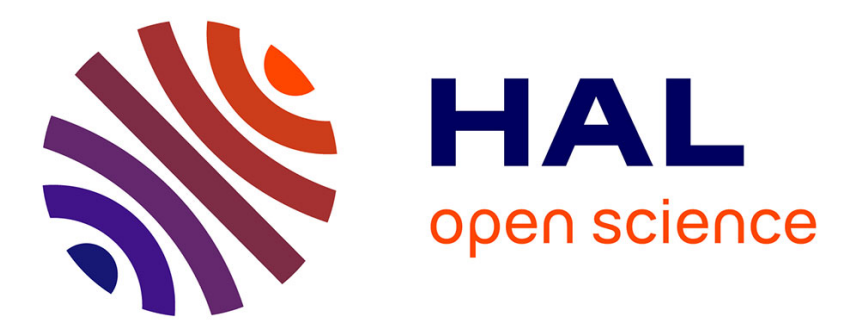

\title{
Overpressure on fingertips prevents state estimation of the pen grip force and movement accuracy
}

Jérémy Danna, Mathilde Nordlund, Didier Louber, Simon Moré, Laurence

Mouchnino

\section{- To cite this version:}

Jérémy Danna, Mathilde Nordlund, Didier Louber, Simon Moré, Laurence Mouchnino. Overpressure on fingertips prevents state estimation of the pen grip force and movement accuracy. Experimental Brain Research, 2022, 240, pp.189-198. hal-03377269

\section{HAL Id: hal-03377269 \\ https://hal.science/hal-03377269}

Submitted on 14 Oct 2021

HAL is a multi-disciplinary open access archive for the deposit and dissemination of scientific research documents, whether they are published or not. The documents may come from teaching and research institutions in France or abroad, or from public or private research centers.
L'archive ouverte pluridisciplinaire $\mathbf{H A L}$, est destinée au dépôt et à la diffusion de documents scientifiques de niveau recherche, publiés ou non, émanant des établissements d'enseignement et de recherche français ou étrangers, des laboratoires publics ou privés. 
Overpressure on fingertips prevents state estimation of the pen grip force and movement accuracy

Jérémy Danna ${ }^{1}$, Mathilde Nordlund ${ }^{1}$, Didier Louber ${ }^{1}$, Simon Moré ${ }^{1}$, \& Laurence Mouchnino ${ }^{1}$

${ }^{1}$ Aix Marseille Univ, CNRS, LNC, Marseille, France

\section{Corresponding author:}

Jérémy Danna

ORCID ID: 0000-0002-3596-880X

CNRS / Aix-Marseille Université

Laboratoire de Neurosciences Cognitives, LNC, UMR 7291, FR 3C 3512,

3 place Victor Hugo,

13331 Marseille cedex 3

Tel.: +33413550956

Fax: $\quad+33413550958$

E-Mail:Jeremy.danna@univ-amu.fr

\section{Acknowledgements}

The authors would like to thank David Wood (English at your Service, www.eays.eu) for revising the English of the paper.

\section{Declarations:}

Funding: This work was supported by the DefiAuton CNRS project and the French National Research Agency ANR grant “COMTACT” (ANR-20-CE28-0010). This work was carried out within the Institut Convergence ILCB (ANR-16-CONV-0002).

Conflicts of interest/Competing: None. 
Availability of data and material: Data are available on request from the corresponding author.

Code availability: Not applicable

Authors' contributions: JD: Conceptualization, Methodology, Data acquisition, Data analysis, Writing- Original draft preparation. MN: Data acquisition, Data analysis. DL: Methodology. SM: Methodology. LM: Conceptualization, Methodology, Data acquisition, Data analysis, Writing- Reviewing and Editing.

Ethics approval: This study was conducted in accordance with the ethical standards set out in the Declaration of Helsinki and approved by the local Ethics Committee

Consent to participate: All participants signed a written informed consent before starting the experiment.

Consent for publication: All co-authors give their consent for this publication. 


\section{Abstract}

We tested the hypothesis that the inability to move a pen accurately in a graphic task is partly due to a decrease of afferent somatosensory information resulting from overpressure on the tactile receptors of the fingers holding the pen. To disentangle the depressed somatosensory origin from an altered motor command, we compared a condition in which the participant actively produces pressure on the pen (active grip) with a condition in which pressure is passively applied (passive grip, no grip-related motor command). We expected that the response of the somatosensory cortex to electric stimulation of the wrist's tactile nerve (i.e., SEP) would be greater in the natural pen grip (baseline condition) than in the two overpressure conditions (actively or passively induced). Fifteen adults were required to trace a geometrical shape in the three grip conditions. The SEP amplitude was not significantly different between the baseline and both overpressure conditions. However, behavioral results showed that drawing accuracy is impaired when the pressure on the pen is increased (passively or actively). Cortical source analyses revealed that the activity of the superior parietal areas (SPL) increased in both overpressure conditions. Our findings suggest that the SPL is critical for sensorimotor integration, by maintaining an internal representation of pen holding. These cortical changes might witness the impaired updating of the finger-pen interaction force for such drawing actions under visual guidance.

Keywords: Graphonomics, Pen Holding, Sensory feedback, Electroencephalography, Somatosensory Evoked Potential 


\section{Introduction}

In graphic tasks, such as drawing or handwriting, biomechanical factors such as grip posture or abnormal pen grasp force are considered as a cause to explain impaired drawing accuracy or handwriting legibility. For example, Schneck (1991) investigated several grip postures (e.g., palmar supinate grip, cross thumb grip or tripod grip) in children with or without handwriting difficulties and observed that children with handwriting difficulties used the traditional tripod grip to a lesser extent as compared to children without handwriting problems. However their observations were not confirmed by more recent studies that failed to find a correlation between pen grip posture and handwriting legibility and/or speed (Koziatek \& Powell, 2003; Shah \& Gladson, 2015; Schwellnus et al., 2012). One can reasonably expect that the handwriting performance is conditioned, to some extent, by the level of force exerted by the fingers on the pen. Thanks to the development of pen prototypes, Chau and colleagues (2006) have shown that children with cerebral palsy writing with their unaffected hand exerted a lower grip force, likely due to a weaker grasp, than healthy children with no handwriting difficulties. Note that the results of this cases study do not clarify whether the weaker grasp or the capability to vary force is at the origin of handwriting difficulties. Indeed, an increase in motor command induces an increase in limb stiffness and may deteriorate writing performance. This can either be caused through co-contraction of the agonist and antagonist muscles engaged in the writing action, as observed in the case of writer's cramp (Hermsdörfer et al., 2011), or can be due to the enslaving effect (i.e., involuntary force production by fingers muscles anatomically linked or separated, Zatsiorsky et al., 2000). This altered motor command resulted from stressful environments (Van Gemmert \& Van Galen, 1997) or was observed in children with neurodevelopmental disabilities (Smits-Engelsman et al., 2003). Although the grip is important because it allows the fine movements necessary for writing, several studies failed to find a consistent relationship between pen grip biomechanical factors and handwriting legibility (e.g., Lin et al., 2017; Parush et al., 1998; Rosenblum et al., 2006).

One of the reasons for this lack of findings might be a confounding aspect between sensory and motor origin of the impaired writing performance. Indeed, increasing the grip stiffness and the forces on the pen could lead to a decrease of the afferent feedback from the fingertips as a consequence of the overpressure exerted on the tactile receptors. Indeed, 
tactile fibers stop firing when subjected to sustained pressure exerted on the skin where the mechanoreceptors are embedded (Johansson and Vallbo, 1983; Knellwolf et al., 2018, see Macefield, 2005 for a review). For instance, slow adapting afferent fibers (Merkel and Ruffini) are known to decrease their instantaneous firing frequency throughout a sustained stimulus (e.g., normal force exerted on the skin surface) while the rapidly adapting type I and II (Meissner and Pacinian corpuscle) afferents only respond to brisk mechanical transients (Knibestöl, 1975; Vedel and Roll, 1982; Vallbo et al., 1995; Trulsson, 2001; Kennedy and Inglis, 2002). Combining brain imaging and behavioral data, a decreased somatosensory transmission was observed, from the periphery to the cortical areas, this was provoked by skin compression on the foot sole when standing, supporting a heavy weight (Handrigan et al., 2012; Lhomond et al., 2016) or when pressure is increased within a small surface area (Fabre et al., 2021).

There is also some evidence of somatosensory online control while writing. For instance, suppressing somatosensory feedback after either transient (Ebied et al., 2004) or permanent (Hepp-Reymond et al., 2009; Danna \& Velay, 2017) somatosensory deafferentation alters writing kinematics. However, the written trace seems to remain roughly legible as it is known to be principally under visual guidance (Danna \& Velay, 2015; Teasdale et al., 1993). These altered kinematics are not surprising since tactile receptors provide information related to the time course, magnitude, direction and spatial distribution of contact forces between the pen and the fingers. The writer encodes information on the physical properties of the pen (shape, texture, weight) to adequately set the grip force in order to prevent the pen slipping out of their fingers. More generally, it is well established that when tactile information is lacking, both grasping and manipulation of objects are altered (for a review, Johansson \& Flanagan, 2009). More precisely, an increase in grip force is observed when tactile sensitivity was reduced (e.g., Johansson \& Westling, 1984; Naceri et al., 2021).

On the basis of the putative function of the somatosensory information and/or of the pen grip biomechanical factors during writing performance, we hypothesize that the cause of movement inaccuracy is not due to a stronger pen grip motor command per se but rather to the associated decrease in afferent tactile information. We will use a protocol that will enable us to decrease sensory information while the pen grip motor command is either present or removed. We will compare the drawing accuracy and the cortical response to electrical tactile 
afferents stimulations occurring with augmented pressure on the pen grip, passively applied to the finger (i.e., no pen grip motor command) or actively generated by the participant. Assuming that this manipulation is effective, we expected drawing accuracy to be altered similarly in both overpressures conditions as compared to the baseline condition (i.e., natural pen grip). The decrease in the somatosensory evoked potentials $\left(P_{50} N_{80} S E P s\right)$ in response to electric stimulation of the tactile afferents is considered as the neural signature of an attenuated sensory processing (Lhomond et al., 2016: Fabre et al., 2021). According to the seminal study of Desmedt and Robertson (1977), the SEP in response to finger stimulation originates, for the early component $(<60 \mathrm{~ms})$, from the primary somatosensory cortex (SI) and is highly representative of the stimulus characteristics (e.g., intensity, frequency). The later cortical responses $(>80 \mathrm{~ms})$ following tactile stimulation emanate from the secondary somatosensory cortex (SII). We predicted that, during the drawing task, the amplitude of the SEP would be smaller when the stimulation occurred with increased grip force on the pen as compared to a natural grip force condition (i.e. baseline condition).

\section{Material and methods}

\subsection{Participants}

Fifteen self-declared right-handed participants (mean of $24 \pm 2$ years old, 12 women) participated in the experiment. In this particular task of drawing, the extensive training of the right hand for writing is the relevant factor to determine handedness. None of them reported that they were ambidextrous. For the experiment, they were required to use their hand they learned to write with. None of the participants reported any relevant medical history. They had normal or corrected-to-normal vision. All participants signed a written informed consent before starting the experiment, in accordance with the ethical standards set out in the Declaration of Helsinki and approved by the local Ethics Committee.

\subsection{Tasks and Materials}

Participants were seated in front of a digitizing tablet fixed onto a table, with the forearm resting on the tablet (Fig.1A). At a Go tone signal, they had to trace precisely and continuously a figure having the shape of an " 8 " in superposition with a model, at constant speed chosen 
by the participants, for 15 seconds (referred to as Drawing task). The model appeared on a sheet of paper fixed to the graphic tablet (Wacom, Intuos Pro, sampling frequency $200 \mathrm{~Hz}$, spatial resolution $0.05 \mathrm{~mm}$ ). The pen was equipped of three Force Sensitive Resistor (FSR) sensors (FSS low profile force sensors FSS1500NSB from Honeywell ${ }^{\mathrm{TM}}$, see Fig. 1A) in order to record the forces exerted by the thumb, the index, and the major in the tripod pencil grip. These sensors provide precise and reliable force sensing performance, with a repeatability at \pm 10 grams at 300 grams (variations less than $4 \%$ ) and a sensitivity at $0.24 \mathrm{mV} / \mathrm{gram}$. We amplified the signal with a gain of 10 and we calculated the sum of the three sensors in Newton (N). Data from the FSR sensors were collected with a fast acquisition system (ADwin Pro $^{\circledR}$, sampling frequency $1000 \mathrm{~Hz}$ ). The force sensors were placed at $45 \mathrm{~mm}$ relative to the pen tip to ensure that they did not interfere with the magnetic field of the tablet, even when tilting the pen. Data from the graphic tablet were collected with a home-made Matlab ${ }^{\circledR}$ program and Psychophysics Toolbox Version 3 (Psychtoolbox-3). Together with the drawing task a control static task was performed (referred to as Static task). The participant held the pen fixed on the figure for 15 seconds. 
A
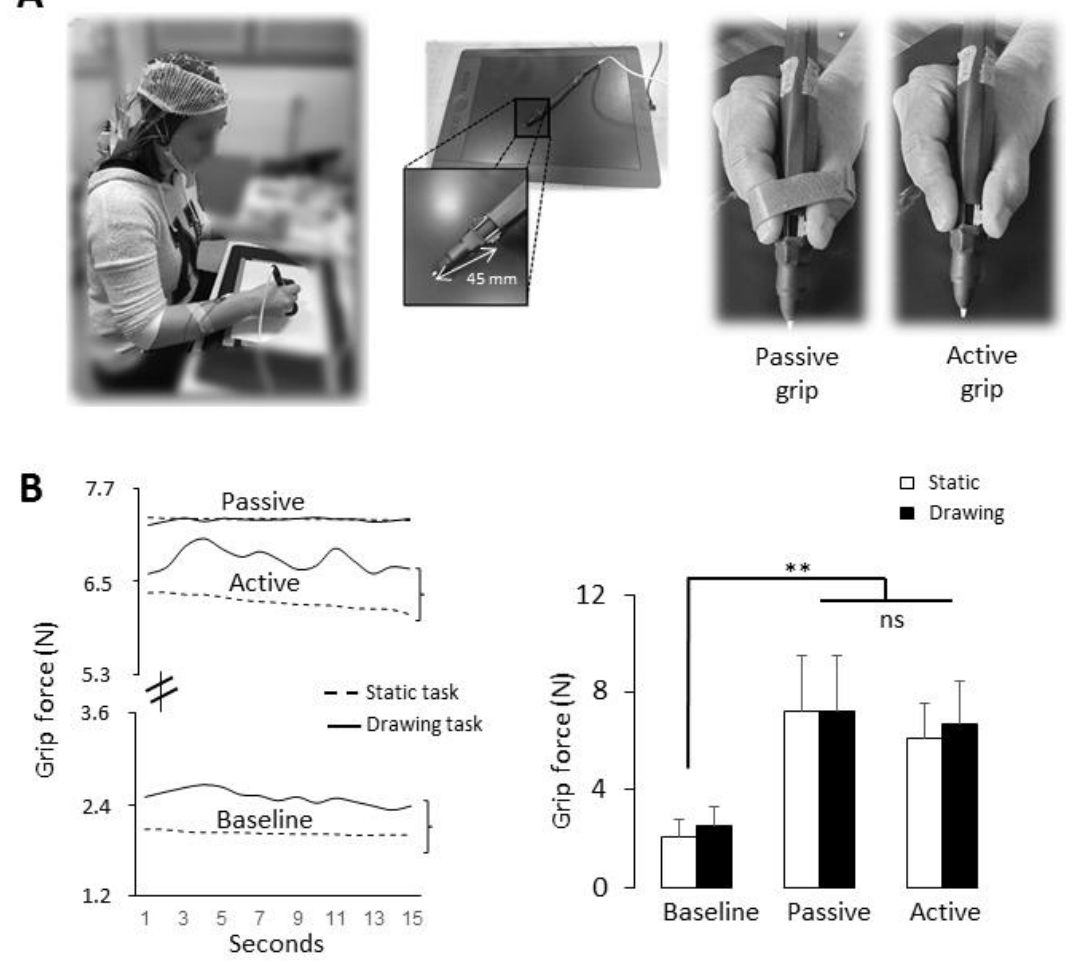

C
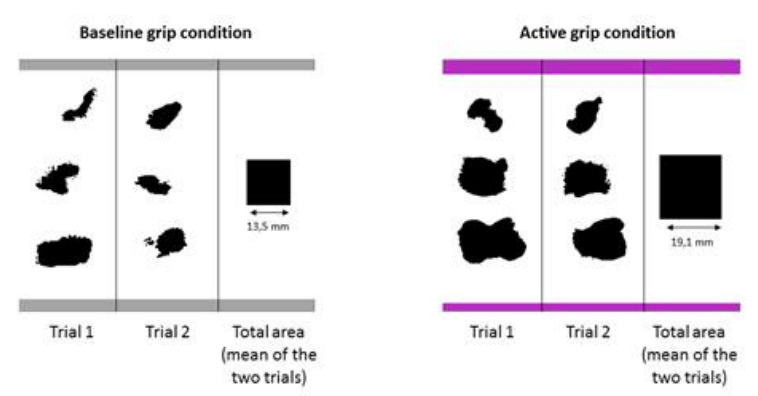

Figure 1. A) Left panel, illustration of the task performed by a participant equipped with an electrocap for EEG recordings, and two electrodes affixed near to the wrist for stimulation of the median nerve. Middle panel, illustration of the graphic tablet and of the pen equipped with three Force Sensitive Resistor (FSR) sensors. Right panel, illustration of the passive and active grip conditions. B) Grip force recorded and averaged at each second during the $15 \mathrm{sec}$ trial duration (left) or averaged over the whole trial (right) for the three grip conditions (Baseline, Passive and Active) in the drawing (drawn line) and static (dotted line) tasks. C) Illustration of the contact area between the digits and the pen for two trials under a Baseline (left) and an Active (right) grip condition in the Static task (in one participant). The contact area was identified with the use of black paint on the fingers and a white sheet wrapped around the pen. Then, the sheet was scanned in black and white with the same resolution to count the black pixels of the figures (same size: 142×290 pixels for the three conditions) with matlab. The black square corresponds to the total area averaged between the two trials in each condition. Note that the contact area under the Active condition is twice the size of that under the Baseline condition. The deformation of the extremity of the fingers is strikingly reminiscent of what was found when normal weight individuals were loaded with external weights. Greater total plantar surface and higher mean plantar pressure were observed (Vela et al., 1998; Wright et al. 2012). This may significantly affect the afferent outflow from the tactile mechanoreceptors as observed in Lhomond et al's (2016) and Fabre et al.'s (2021) studies. 
Three pen grip conditions were tested in both drawing and static tasks. In the Baseline grip, the participant was asked to hold the pen in a natural and spontaneous manner. In the Active grip, the participant was asked to significantly increase the pen grip force and to maintain it constant during the entire trial. In the Passive grip, the participants' fingers were tied tightly onto the pen with a self-adhesive adjustable band in such a way that the total forces exerted by the fingers on the pen was comparable to the Active grip. Before the passive condition, the experimenter had a visual feedback of the force applied on the sensors to verify that the forces exerted in the passive and active conditions were similar. Active and passive grip conditions are illustrated in Fig. 1A (right panel). The three pen grip conditions were counterbalanced between participants. Each pen grip condition was tested over 10 Drawing trials and 10 Static trials (randomized within each pen grip condition).

\subsection{Behavioral recordings and analyses}

The axial pressure exerted by the pen on the tablet was recorded from the graphic tablet (resolution 2048 levels of pressure) that we converted in Newton after calibration of the pen on a weighting scale. The results of the calibration are illustrated in Figure $2 \mathrm{~A}$. As can be seen, the values differ between the upward calibration (where the weight increased during the calibration - in blue) and the downward calibration (where the weight decreased -in red). Thus, we considered the averaged values between the two calibration conditions for converting the pen pressure data in Newton in each condition for each participant. 
A

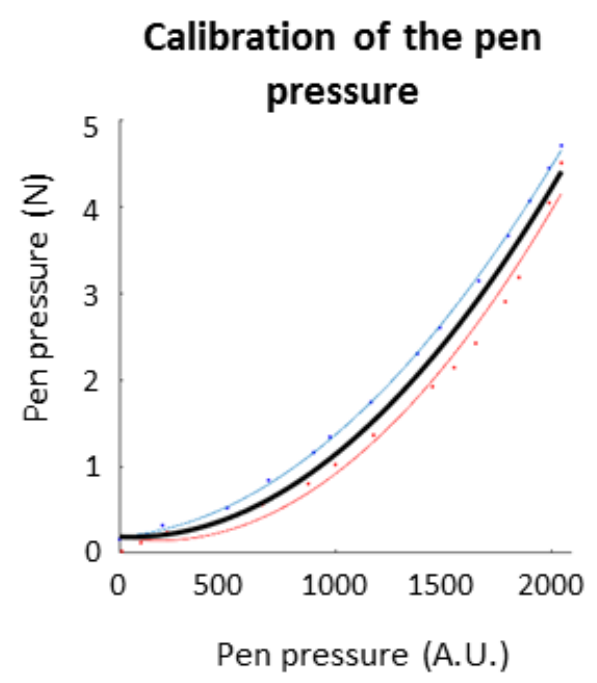

C

Drawing path

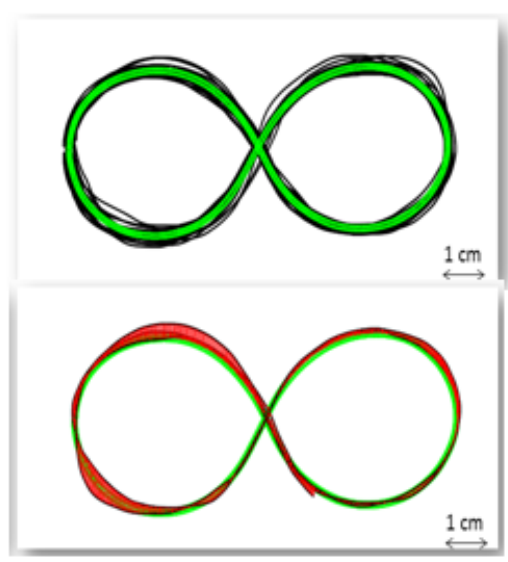

B

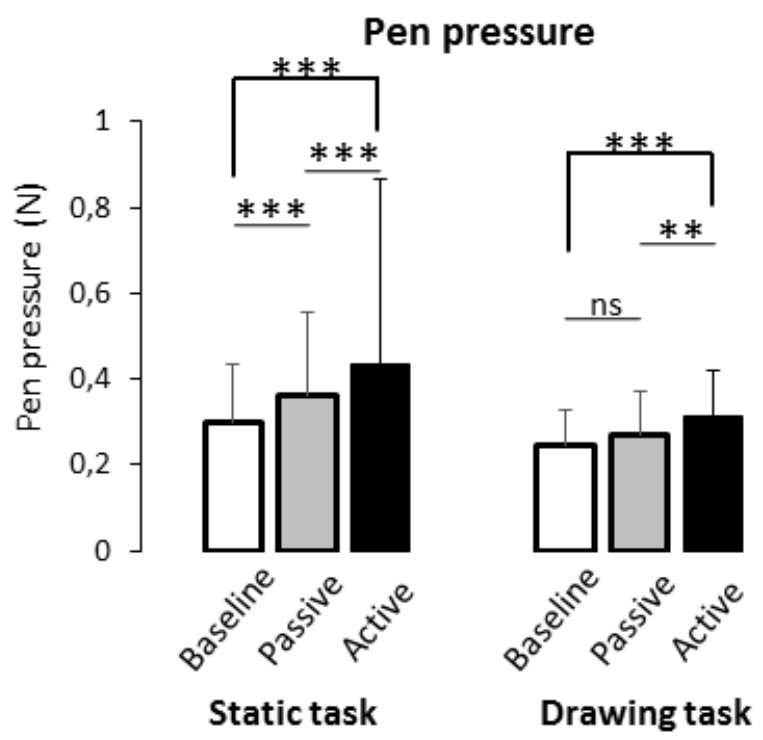

D

\section{Spatial error}

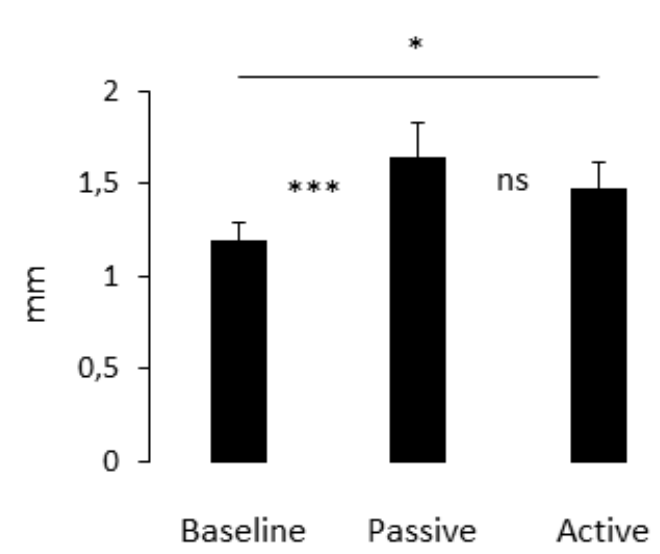

Figure 2. A) Relationship between pen pressure recorded with a weighting scale (in N) and pen pressure recorded by the tablet (in arbitrary unit - A.U.). In blue: data from the upward calibration (the weight increased during the calibration); in red: data from the downward calibration (the weight decreased during the calibration); in black: averaged data between the upward and downward calibration. B) Mean pen pressure under the Baseline, Passive, and Active grip conditions during the Static (left) and Drawing (right) tasks. Error bars correspond to standard error across participants. ${ }^{*} p$ $\left.<0.05 ;{ }^{* *} p<0.01 ;{ }^{* * *} p<0.001\right)$. C) Illustration of the method used for computing spatial error in the drawing trials. Top: illustration of the theoretical shape (green) as defined through the mean coordinates of the produced shapes (black) on a cycle-by-cycle basis for a subject in the Baseline grip condition (step 1). Bottom: illustration of the Euclidian distance (in red) between the produced shape and the theoretical shape for the same subject (step 2). D) Mean spatial error during the drawing task under the three grip conditions (Baseline, Passive and Active). Error bars correspond to standard error across participants. ( $p<0.05 ; * * *<0.001)$. 
Furthermore, in the drawing task, the pen coordinates were also recorded from the tablet to compute the mean tangential velocity $(\mathrm{mm} / \mathrm{s})$ and a spatial error index. This spatial index was computed in two steps (for an illustration, see Fig. 2C). In the first step, a theoretical shape (in green) was defined through the mean coordinates determined on a cycle-by-cycle basis (step 1, top panel in Fig. $2 \mathrm{C}$ ). In the second step, the spatial error was defined through the mean Euclidian distance between the observed data in each condition and the theoretical shape provided to each participant. This distance is illustrated in red in Figure $2 \mathrm{C}$ (bottom panel). A 13-seconds window excluding the first and last seconds was analyzed in order to exclude any acceleration and deceleration that can appear at the beginning or the end of each trial.

\subsection{Stimulation procedure}

Fourteen stimulations were electrically delivered to the median nerve at the wrist during static and drawing tasks. Each stimulation was separated by 1 second. All stimulations were analyzed (total of 140 stimulations in each condition and task). These stimulations were delivered by a DS5 isolated bipolar constant current stimulator. Each stimulation consisted of a single rectangular $10 \mathrm{~ms}$ pulse. The stimulation intensity was determined as follows: for each participant while their arm was in a relaxed position, we first found the lowest intensity which resulted in a constant perception of the stimulation (mean amplitude: $0.93 \pm 0.25 \mathrm{~mA}$ ). This stimulation was determined as the baseline value. The stimulation intensity was then set at $25 \%$ higher than the baseline value for each participant. For all participants, this intensity was below the motor threshold.

\subsection{Electrophysiological recordings and analyses}

Electroencephalographic (EEG) activity was continuously recorded from $64 \mathrm{Ag} / \mathrm{AgCl}$ surface electrodes embedded in an elastic cap (BioSemi ActiveTwo system: BioSemi, Netherlands). The signal was pre-amplified at the electrode sites and digitized at a sampling rate of $1024 \mathrm{~Hz}$. Off-line filtering with a $50 \mathrm{~Hz}$ digital notch filter and a $0.5-45 \mathrm{~Hz}$ band-pass digital filter (48 dB/octave) implemented by BrainVision Analyzer 2 software (Brain Products, Germany) was applied. SEPs were obtained by averaging, for each participant, task and condition, all 
synchronized epochs relative to the electrical stimulus. The average amplitude of the $50 \mathrm{~ms}$ pre-stimulus epoch served as baseline. We measured the SEPs over the C3 electrode as this electrode overlays the sensorimotor cortices, particularly the representation of the right hand on the homunculus. The earliest discernible positive $\left(P_{50}\right)$ and negative $\left(N_{80}\right)$ peaks following each stimulus were identified. The amplitude of the $\mathrm{P}_{50} \mathrm{~N}_{80}$ waveform was measured peak-topeak (Fig. 3A).

The sources of the cortical activities were reconstructed with Brainstorm software (Tadel et al., 2011), freely available at http://neuroimage.usc.edu/brainstorm) with the preprocessed averaged data. We employed the minimum-norm technique to resolve the inverse problem with unconstrained dipole orientations. The forward model was computed for each condition using a boundary element method (BEM) on the anatomical MRI Colin 27 high-resolution brain template (330 117 vertices), a predominant volume conductor model from the Montreal Neurological Institute (Mosher et al., 1999; Huang et al., 2016). 


\section{A Somatosensory evoked potential}

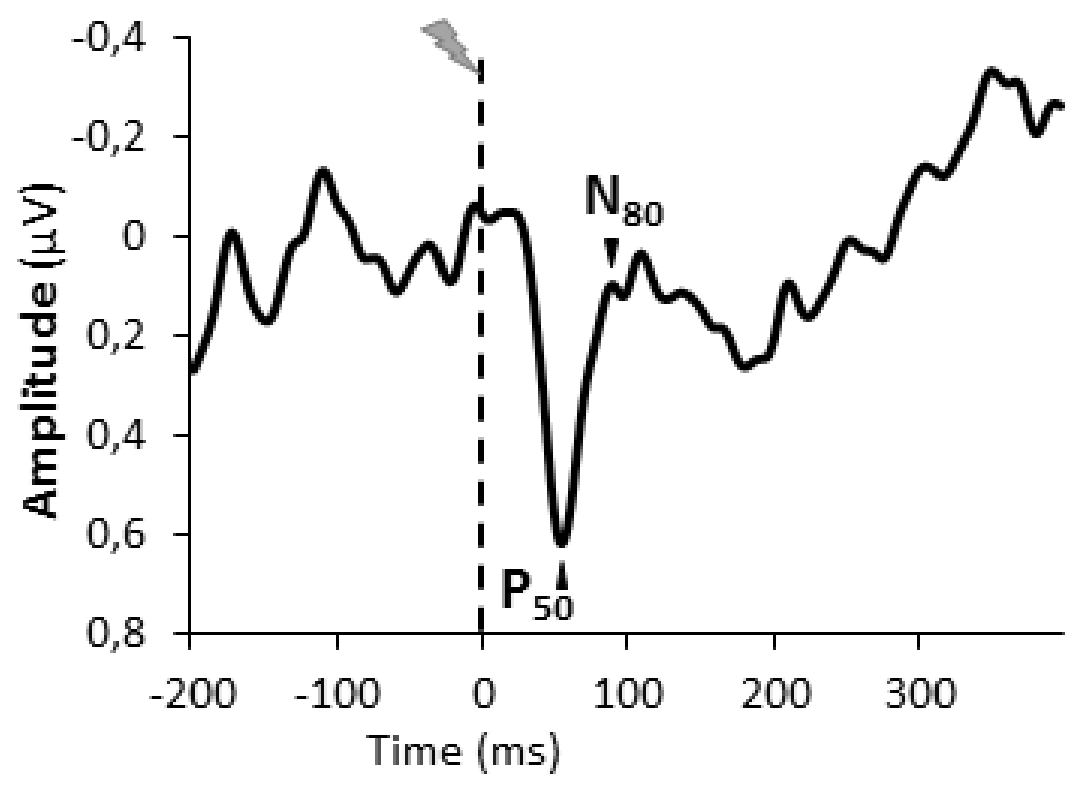

B

$\mathrm{P}_{50} \mathrm{~N}_{80}$ amplitude

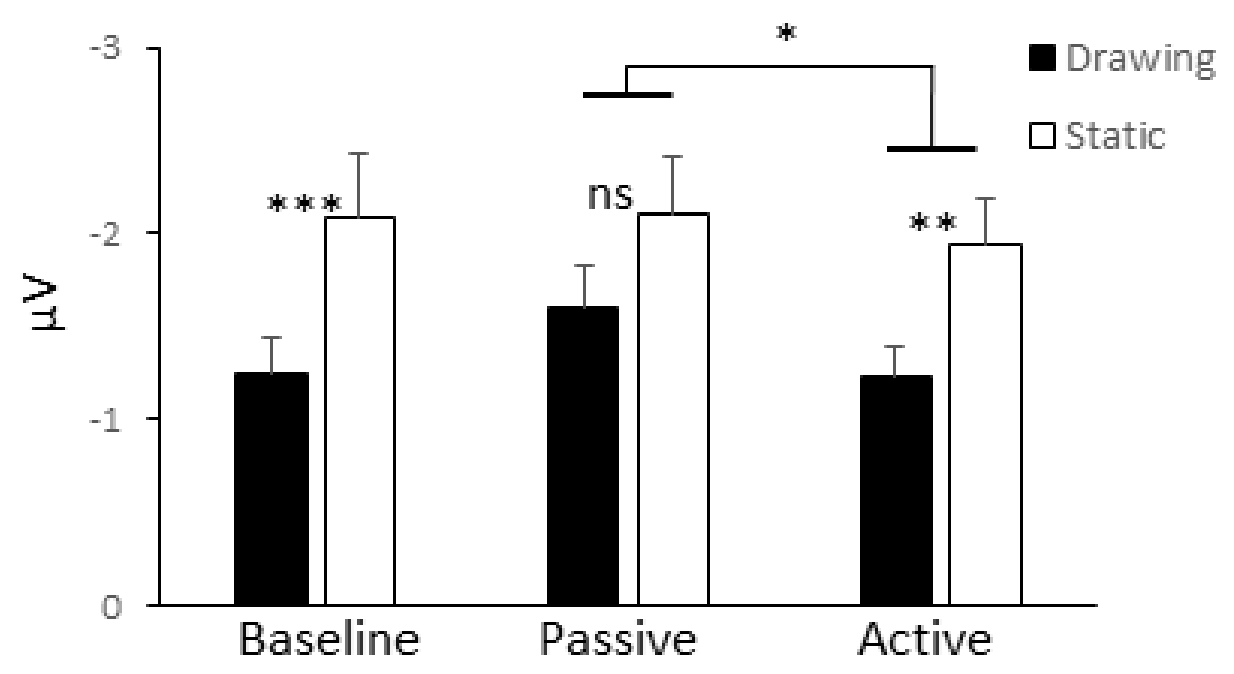

Figure 3. A) Grand average SEP for all participants recorded at C3 under the baseline grip condition. B) Mean for the 140 stimulations of the SEP amplitude. Error bars correspond to standard error across participants. ( $p<0.05 ;{ }^{* *} p<0.01 ;{ }^{* *} p<0.001$ ). 


\subsection{Statistics}

A repeated measures ANOVA was conducted with the two 'Tasks' (Drawing and Static) and the three 'Grip' conditions (Baseline, Passive, and Active) for all variables, except for spatial accuracy and movement velocity that were related to the performance in the Drawing task only. Furthermore, the relationship between grip force and downward pressure on the tablet was tested with Pearson's correlation tests (on the averaged data per condition and per participant). All significance thresholds were set to $p<0.05$. Tukey's HSD post hoc tests were applied when necessary.

\section{Results}

\subsection{Motor performance}

To assess whether participants complied with the force requirement during the trial, the force of each finger (i.e. thumb, index, and major) was measured and averaged every second during the trial duration, then averaged for each trial (Fig. 1B, left panel). The mean grip force of each participant was submitted to a repeated measures ANOVA with the 'Grip' conditions (Baseline, Passive and Active) and the 'Task' (Drawing and Static). As expected (Fig. $1 B$, right panel), the ANOVA revealed a main grip condition effect $\left(F_{2,28}=77.11, p<0.001, \eta^{2} p\right.$ $=0.85)$. Tukey's HSD post hoc test showed that Active and Passive grip conditions were about three times higher than the Baseline condition ( $p<0.001$ for both comparisons) and that the overpressures actively generated on the pen were comparable to those passively applied $(p=$ $0.17, \mathrm{NS})$. In addition, the absence of modulations across the drawing trial in Passive condition as compared to both Active and Baseline conditions suggested that in the Passive condition no pen grip motor command was present (Fig.1B, left panel). Furthermore, we observed a $200 \%$ increase in contact surface area with increased grip force (for an illustration, see Fig. $1 \mathrm{C}$ and caption for commentary).

Variables related to motor performance were analyzed to verify whether drawing performance varied between the pen grip conditions. Overall, the results showed that drawing performance was slightly affected by the different conditions of grip force. The spatial error was below $2 \mathrm{~mm}$. Nevertheless, the ANOVA revealed a main effect of Grip on spatial error $\left(F_{2}\right.$, $28=8.85, p=0.001$, Fig.2D). Post-hoc analysis showed that the spatial error was greater in 
Passive and Active grip conditions than in the Baseline $(p<0.001$ and $p<0.05$ for Passive and Active conditions, respectively) with no difference between the two increased grip force conditions $(p=0.29)$. The changes in accuracy in these conditions were not due to a faster drawing performance as the mean velocity did not reveal a main effect of Grip $\left(F_{2,28}=1.009\right.$; $p=0.37$; overall mean: $83 \pm 52 \mathrm{~mm} / \mathrm{s}$ ).

To further investigate whether the pen downward pressure on the tablet changes with the increased grip force, we analyzed the force exerted on the tablet (Fig. 2B). The results showed a main effect of Task $\left(F_{1,14}=9.54 ; p<0.01\right)$, a main effect of Grip condition $\left(F_{2,28}=\right.$ $11.02, p<0.001)$, and a significant interaction $\left(F_{2,28}=8.58, p=0.01\right)$. When comparing the tasks, it is not surprising to note that the downward pressure on the tablet was lower when drawing than when the pen remained static. This is likely due to the need to slide the pen across the tablet to comply with the drawing task. Interestingly, the post hoc analysis of the Task by Grip interaction revealed that, in the Drawing task, the pressure exerted on the tablet was higher in the Active condition than in both Baseline $(p<0.001)$ and Passive $(p<0.001)$ conditions which did not differed $(p=0.36, N S)$. In the static task, the pen downward pressure differed between the three grip conditions ( $p<0.001$ for the three comparisons). The Pearson's correlation tests between pen grip force and downward pressure revealed a significant correlation in the Baseline conditions $(R=0.56$ in both the Static and Drawing tasks). In the Passive and Active grip conditions, the analysis revealed that these two variables are no longer correlated $(\mathrm{R}<0.25, \mathrm{NS})$.

\subsection{Brain activity}

To assess whether somatosensory processes were disrupted due to increased grip force, we analyzed the $\mathrm{P}_{50} \mathrm{~N}_{80}$ SEP amplitudes (Fig. 3B). The results showed a main effect of grip condition $\left(F_{2,28}=3.31 ; p<0.05\right)$. Post hoc analyses showed that the SEP amplitude was smaller in the Active condition as compared to the Passive condition $(p=0.04)$. Both Active $(1.59 \pm$ $0.66 \mu \mathrm{V})$ and Passive $(1.87 \pm 0.89 \mu \mathrm{V})$ conditions did not differed from the Baseline condition $(1.69 \pm 0.95 \mu \mathrm{V})(p=0.23$ and $p=0.66$ for Passive and Active conditions, respectively).

It is not surprising to note a main effect of Task $\left(F_{1,14}=16.66 ; p=0.0011\right)$. Post hoc analyses showed that the $\mathrm{P}_{50} \mathrm{~N}_{80}$ amplitude was smaller in the drawing task than when the participants 
held the pen static in both the Baseline $(p=0.001)$ and Active $(p<0.01)$ conditions. In the Passive condition the $\mathrm{P}_{50} \mathrm{~N}_{80}$ amplitude did not differ significantly between the Drawing and Static tasks $(p=0.09)$. Overall, these results suggest that the well-known general sensory suppression caused by the motor command per se, was present for both self-generated pengrip force conditions (Baseline and Active) but not significantly for the Passive pen grip.

No task or grip effects were observed on the $P_{50}$ latency (overall mean $52 \pm 8 \mathrm{~ms}$ ) and $\mathrm{N}_{80}$ latency (overall mean $\left.80 \pm 9 \mathrm{~ms}\right)\left(F_{1,14}=0.58 ; p=0.45\right.$ and $F_{2,28}=0.82 ; p=0.44$ for task and grip effect for the $P_{50}$ and $F_{1,14}=0.31 ; p=0.58$ and $F_{2,28}=1.74 ; p=0.19$ for task and grip effect for the $\mathrm{N}_{80}$ ).

To investigate the brain activation changes during and after the $\mathrm{P}_{50} \mathrm{~N}_{80}$ SEP we computed brain activity (Fig. 4). As shown in Figure 4A, during the $\mathrm{P}_{50} \mathrm{~N}_{80}$ SEP, the source analyses revealed an increase activation of the premotor areas (PMd and SMA) contralateral to the drawing movement in the Active relative to Baseline condition. No such increase in activation was observed when comparing Passive and Baseline conditions (Fig. 4B). Later starting at about $80 \mathrm{~ms}$, the statistical source maps revealed consistent activation of the superior lobules (SPL, BA 7) of the parietal cortex. The increase SPL activity was observed in both overpressure pen grip conditions (Active and Passive) as compared to Baseline (Fig 4A and B). This suggested that the increase in activity was impervious to self-generated pen grip as it was observed in the Passive condition but was rather related to the skin compression of the fingers on the pen. It was noteworthy that SPL increase activation is associated with increase activation of the primary somatosensory (S1) in the Active condition (Fig. 4A) and to the increase activation of the dorsal part of the posterior cingulate cortex in the Passive condition (Fig. 4B). 


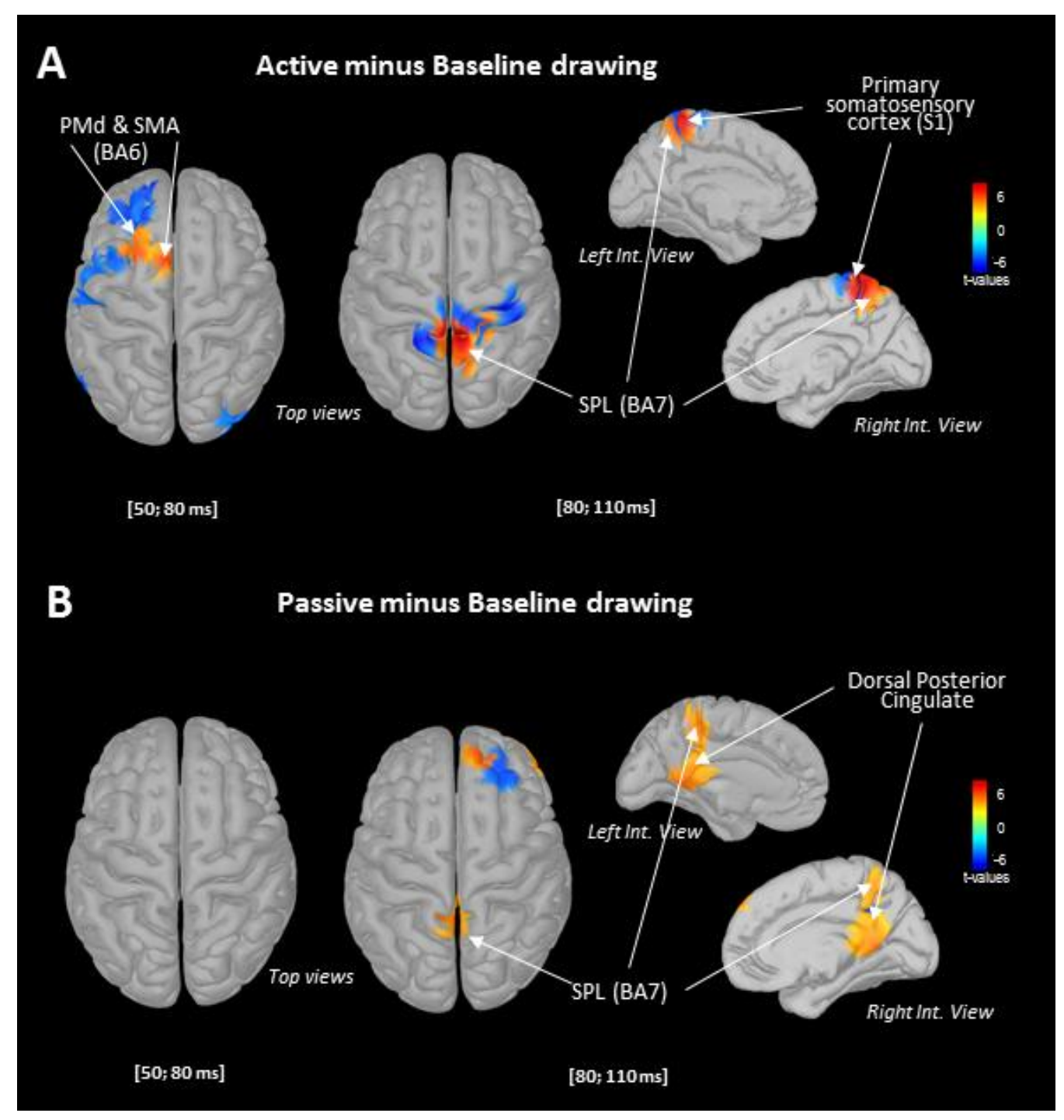

Figure 4. Statistical source estimation maps for contrasts [Active minus Baseline] (top) and [Passive minus Baseline] (bottom) for the drawing task. Significant $t$-values $(p \leq 0.05, \mathrm{n}=15)$ of the source localization were shown at different timing points to assess the dynamics of these activities.

\section{Discussion}

In this study, we tested the hypothesis that degraded sensory feedback with or without a motor command for pen grip decreases the accuracy of a drawing task. Our behavioral results (i.e. spatial error) showed that accuracy is impaired at least slightly when the pen grip force is increased (passively or actively). The lack of accuracy in the Drawing task was not related to the depression of the $\mathrm{P}_{50} \mathrm{~N}_{80} \mathrm{SEP}$, which could have witnessed a decrease in tactile afferents transmission. Rather it was associated with a late increase activation of the SPL, which could be an indicator of difficulty in providing an internal estimate of the grip force during the drawing task. 


\section{Ruling out depressed stimulus-evoked activity}

The main factor that argues against depressed sensory processes as an explanation for the current behavioral findings, is the SEP-motor command relationship. The depressed transmission of the tactile inputs was not the source of the drawing inaccuracy when greater force was applied to the pen for both Active and Passive conditions. Rather the $\mathrm{P}_{50} \mathrm{~N}_{80} \mathrm{SEP}$ reflects the well-known sensory gating mechanism because it occurs in both self-generated pen grip conditions with different amounts of grip force (Baseline and Active condition) for the Drawing task relative to the Static task. This centrally driven attenuation of afferent input occurs if the internal prediction of the action's sensory consequences is similar to the sensory input obtained during movement execution (Seki et al., 2003; Voss et al., 2006; Williams \& Chapman, 2002). In line with Von Holst's \& Mittelstaedt's seminal work (1950), an internal model of the sensory consequences of active pen grip is used to selectively suppress tactilekinesthetic reafference. This sensory suppression of grip-induced feedback is considered as a neural mechanism that allows the central nervous system to distinguish sensory inputs that arise from external sources from inputs resulting from self-generated movements (Blakemore, Wolpert, \& Frith, 1998). Conversely, the sensory attenuation disappeared in the Passive condition because of the unpredicted tactile feedback due to the passive skin compression of the participants' fingers, tightly attached to the pen. Note that in the Passive condition, the tactile inputs came from two distinct areas, between the fingers and the pen and between the fingers and the band. While this double passive skin compression did not change the amplitude of the SEP in the Static task, it could have interfered with the processing of tactile information in the Drawing task.

\section{Less efficient stimulus processing in maintaining internal representation of the grip force}

A clear result from our study was that the lack of accuracy in drawing performance was associated with greater activation of the superior parietal lobule (SPL) starting about $80 \mathrm{~ms}$ after the stimulation in both Active and Passive conditions relative to Baseline. Increasing the force of the pen grip seems to result in a compensatory recruitment of sensory integrative mechanisms evidenced by the SPL activation, which may help to cope with changed tactile 
feedback. Indeed, the posterior parietal cortex (PPC) and in particular the SPL is hypothesized to maintain an internal estimate of limb state, which is continually updated in accordance with changing inputs, for example during actions and their preparation (Wolpert, Goodbody, \& Husain, 1998; Barany et al., 2014). The convergence of tactile inputs to the SPL by direct thalamocortical projections (Pearson, Brodal, \& Powell, 1978; Pons \& Kaas, 1985; Padberg et al., 2009) or indirect projections via cortico-cortical connections (Friedman et al., 1986; Cavada \& Goldman-Rakic, 1989) allows for this possibility. During the drawing task, the SPL exhibited greater activity with increased pen grip force relative to baseline. This may reflect greater reliance on predictive feedforward control when there is low confidence in relying on sensory cues (here low tactile transmission) (Macuga \& Frey, 2014).

Note that prior to the late involvement of the SPL an early increase in activity of the PMd and SMA in the left hemisphere was observed solely in the Active condition. This is consistent with the presence of an increased motor command and a grip force scaling in the active force condition compared to the baseline condition (White et al., 2013). Furthermore, the late SPL increase in activity is associated with increased activity of S1 solely in the Active condition and with the dorsal posterior cingulate in the Passive condition. Our results are in line with Ruby and Decety's study (2001) in which third-person perspective was compared to first-person perspective during action simulation. The authors showed that the first-person perspective (here the Active condition) resulted in strong activation of the parietal lobule, as well as increased activation in the somatosensory cortex that could witness the programming of the self's movements that can be transformed into execution. By contrast, the third person perspective (here Passive condition) involved the posterior cingulate cortex together with the parietal cortex, which may account for a neural mechanism in the determination of agency. The processing of self-referential stimuli in cortical midline structures, such as the posterior cingulate cortex, is a fundamental component in generating a model of the self (Northoff \& Bermpohl, 2004).

\section{Conclusion}

Finally, our behavioral observation of a lack of correlation between pen grip force and downward force observed in case of overpressure, whether Active or Passive (but not in natural pen grip condition) argued for the lack of an accurate representation of the pen 
position and interaction with the surface. Taken together, the findings of the present study demonstrate a lack of accuracy in drawing performance caused by overpressure on the pengrip. Tactile-kinesthetic inputs processing rather than motor processes is at the origin of the drawing inaccuracy as it is observed in both Passive (i.e., no motor command for the pen-grip) and Active overpressure conditions. The activation of the SPL emerges over the same time course under both Active and Passive conditions, suggesting a failure to maintain an internal representation of the finger-pen position relative to the surface where the drawing is performed, this being necessary for both perception and action.

\section{References}

Barany DA, Dell-Maggiore V, Viswanathan S, Cieslak M, Grafton ST (2014) Feature interactions enable decoding of sensorimotor transformations for goal-directed movement. J Neurosci 34:6860-6873. https://doi.org/10.1523/JNEUROSCI.5173-13.2014

Blakemore SJ, Wolpert DM, Frith CD (1998) Central cancellation of self-produced tickle sensation. Nat Neurosci 1:635-640. https://doi.org/10.1038/2870

Cavada C, Goldman-Rakic PS (1989) Posterior parietal cortex in rhesus monkey: II. Evidence for segregated corticocortical networks linking sensory and limbic areas with the frontal lobe. J Comp Neurol 287:422-45. https://doi.org/10.1002/cne.902870403

Chau T, Ji JP, Tam C, Schwellnus H (2006) A novel instrument for quantifying grip activity during handwriting. Arch Phys Med Rehab 87:1542-1547.

https://doi.org/10.1016/i.apmr.2006.08.328

Danna J, Velay JL (2015) Basic and supplementary sensory feedback in handwriting. Front Psychol 6:169. https://doi.org/10.3389/fpsyg.2015.00169

Danna J, Velay JL (2017) On the Auditory-Proprioception Substitution Hypothesis: Movement Sonification in Two Deafferented Subjects Learning to Write New Graphic Patterns. Front Neurosci 11:137. https://doi.org/10.3389/fnins.2017.00137

Desmedt JE, Robertson D (1977) Differential enhancement of early and late components of the cerebral somatosensory evoked potentials during forced-paced cognitive tasks in man. J Physiol 271:761-782. https://doi.org/10.1113/iphysiol.1977.sp012025 
Ebied AM, Kemp GJ, Frostick SP (2004) The role of cutaneous sensation in the motor function of the hand. J Orthop Res 22:862-866. https://doi.org/10.1016/i.orthres.2003.12.005

Fabre M, Antoine M, Germain Robitaille M, Ribot-Ciscar E, Ackerley R, Aimonetti JM, Chavet P, Blouin J, Simoneau M, Mouchnino L (2021) Large postural sways prevent foot tactile information from fading: neurophysiological evidence. Cereb Cortex Comm 2:1-10. https://doi.org/10.1093/texcom/tgaa094

Friedman DP, Murray EA, O'Neill JB, Mishkin M (1986) Cortical Connections of the Somatosensory Fields of the Lateral Sulcus of Macaques: Evidence for a Corticolimbic Pathway for Touch. J Comp Neurol 252:323-47. https://doi.org/10.1002/cne.902520304 Handrigan GA, Berrigan F, Hue O, Simoneau M, Corbeil P, Tremblay A, Teasdale N (2012) The effects of muscle strength on center of pressure-based measures of postural sway in obese and heavy athletic individuals. Gait Posture 35:88-91.

https://doi.org/10.1016/j.gaitpost.2011.08.012

Hepp-Reymond MC, Chakarov V, Schulte-Mönting J, Huethe F, Kristeva R (2009) Role of proprioception and vision in handwriting. Brain Res Bull 79:365-370.

https://doi.org/10.1016/j.brainresbull.2009.05.013

Hermsdörfer J, Marquardt C, Schneider AS, Fürholzer W, Baur B (2011) Significance of finger forces and kinematics during handwriting in writer's cramp. Hum Mov Sci 30:807-817. https://doi.org/10.1016/i.humov.2010.04.004

Huang Y, Parra LC, Haufe S (2016) The New York head-a precise standardized volume conductor model for EEG source localization and tES targeting. Neuroimage 140:150-162. https://doi.org/10.1016/i.neuroimage.2015.12.019

Johansson RS, Flanagan JR (2009) Coding and use of tactile signals from the fingertips in object manipulation tasks. Nat Rev Neurosci 10:345-359. https://doi.org/10.1038/nrn2621 Johansson RS, Vallbo AB (1983) Tactile sensory coding in the glabrous skin of the human hand. Trends Neurosci 6:27-32. https://doi.org/10.1016/0166-2236(83)90011-5

Johansson RS, Westling G (1984) Roles of glabrous skin receptors and sensorimotor memory in automatic control of precision grip when lifting rougher or more slippery objects. Exp Brain Res 56: 550-564. https://doi.org/10.1007/BF00237997 
Kennedy PM, Inglis T (2002) Distribution and behaviour of glabrous cutaneous receptors in the human foot sole. J Physiol 538: 995-1002. https://doi.org/0.1113/iphysiol.2001.013087 Knellwolf TP, Burton AR, Hammam E, Macefield VG (2018) Microneurography from the posterior tibial nerve: a novel method of recording activity from the foot in freely standing humans. J Neurophysiol 120: 953-959. https://doi.org/10.1152/in.00937 Knibestöl M (1975) Stimulus-response functions of slowly adapting mechanoreceptors in the human glabrous skin area. J Physiol (Lond) 245: 63-80.

https://doi.org/10.1113/iphysiol.1975.sp010835

Koziatek, S.M., \& Powell, N. J. (2003). Pencil grips, legibility, and speed of fourth-graders' writing in cursive. American Journal of Occupational Therapy, 57, 284-288.

https://doi.org/10.5014/ajot.57.3.284

Lhomond O, Teasdale N, Simoneau M, Mouchnino L (2016) Neural consequences of increasing body weight: Evidence from somatosensory evoked potentials and the frequencyspecificity of brain oscillations. Front Hum Neurosci 10:318.

https://doi.org/10.3389/fnhum.2016.00318

Lin YC, Chao YL, Wu SK, Lin HH, Hsu CH, Hsu HM, Kuo LC (2017) Comprehension of handwriting development: Pen-grip kinetics in handwriting tasks and its relation to fine motor skills among school-age children. Aust Occup Ther J 64:369-380.

https://doi.org/10.1111/1440-1630.12393

Macefield VG (2005) Physiological characteristics of low-threshold mechanoreceptors in joints, muscle and skin in human subjects. Clinic Exp Pharmacol physiol 32:135-144.

https://doi.org/10.1111/j.1440-1681.2005.04143.x

Macuga KL, Frey SH (2014) Differential contributions of the superior and inferior parietal cortex to feedback versus feedforward control of tools. Neurolmage 92:36-45.

https://doi.org/10.1016/j.neuroimage.2014.01.024

Mosher JC, Leahy RM, Lewis PS (1999) EEG and MEG: forward solutions for inverse methods. IEEE Trans Biomed Eng 46:245-259. https://doi.org/10.1109/10.748978 
Naceri A, Gultekin YB, Moscatelli A, Ernst MO (2021) Role of Tactile Noise in the Control of Digit Normal Force. Front Psychol 12:612558. https://doi.org/10.3389/fpsyg.2021.612558 Northoff G, Bermpohl F (2004) Cortical midline structures and the self. Trends Cogn Sci 8:102-107. https://doi.org/10.1016/i.tics.2004.01.004

Padberg J, Cerkevich C, Engle J, Rajan AT, Recanzone G, Kaas J, Krubitzer L (2009) Thalamocortical Connections of Parietal Somatosensory Cortical Fields in Macaque Monkeys are Highly Divergent and Convergent. Cereb Cortex 19:2038-2064.

\section{https://doi.org/10.1093/cercor/bhn229}

Parush S, Levanon-Erez N, Weintraub N (1998) Ergonomic factors influencing handwriting performance. Work 11:295-305. https://doi.org/10.3233/WOR-1998-11306

Pearson RC, Brodal P, Powell TP (1978) The projection of the thalamus upon the parietal lobe in the monkey. Brain Res 144:143-148. https://doi.org/10.1016/0006-8993(78)90440-7 Pons TP, Kaas JH (1985) Connections of area 2 of somatosensory cortex with the anterior pulvinar and subdivisions of the ventroposterior complex in macaque monkeys. J Comp Neurol 240:16-36. https://doi.org/10.1002/cne.902400103

Rosenblum S, Goldstand S, Parush S (2006) Relationships among biomechanical ergonomic factors, handwriting product quality, handwriting efficiency, and computerized handwriting process measures in children with and without handwriting difficulties. Am J Occup Ther 60:28-39. https://doi.org/10.5014/ajot.60.1.28

Ruby P, Decety J (2001) Effect of subjective perspective taking during simulation of action: A PET investigation of agency. Nat Neurosci 4:546-550. https://doi.org/10.1038/87510

Schwellnus,H., Carnahan, H., Kushki, A., Polatajko, H., MissiunaC., \& Chau, T. (2012). Effect of pencil grasp on the speed and legibility of handwriting in children. American Journal of Occupational Therapy, 66, 718-726. https://doi.org/10.5014/ajot.2012.004515

Shah, L. J., \& Gladson, B.L. (2015). The relationship of pencil grasp on college students' handwriting speed and legibility. Journal of Occupational Therapy, Schools \& Early Intervention, 8, 180-191. https://doi.org/10.1080/19411243.2015.1040673

Schneck CM (1991) Comparison of pencil-grip patterns in first graders with good and poor writing skills. Am J Occup Ther 45:701-706. https://doi.org/10.5014/ajot.45.8.701 
Seki K, Perlmutter SI, Fetz EE (2003) Sensory input to primate spinal cord is presynaptically inhibited during voluntary movement. Nat Neurosci 6:1309-1316.

\section{https://doi.org/10.1038/nn1154}

Smits-Engelsman BCM, Wilson PH, Westenberg Y, Duysens J (2003) Fine motor deficiencies in children with developmental coordination disorder and learning disabilities: An underlying open-loop control deficit. Hum Mov Sci 22:495-513.

https://doi.org/10.1016/i.humov.2003.09.006

Tadel F, Baillet S, Mosher JC, Pantazis D, Leahy RM (2011) Brainstorm: A User-Friendly Application for MEG/EEG Analysis. Comput Intel Neurosci 879716.

https://doi.org/10.1155/2011/879716

Teasdale N, Forget R, Bard C, Paillard J, Fleury M, Lamarre Y (1993) The role of proprioceptive information for the production of isometric forces and for handwriting tasks. Acta Psychol 82:179-191. https://doi.org/10.1016/0001-6918(93)90011-F Trulsson M (2001) Mechanoreceptive afferents in the human sural nerve. Exp Brain Res 137(1): 111-116. https://doi.org/10.1007/s002210000649

Vallbo AB, Olausson H, Wessberg J, Kakuda N (1995) Receptive field characteristics of tactile units with myelinated afferents in hairy skin of human subjects. J Physiol 483: 783-795. https://doi.org/10.1113/iphysiol.1995.sp020622

Van Gemmert ARA, van Galen GP (1997) Stress, neuromotor noise, and human performance: A theoretical perspective. J Exp Psychol Human 23:1299-1313.

https://doi.org/10.1037/0096-1523.23.5.1299

Vedel JP, Roll JP (1982) Response to pressure and vibration of slowly adapting cutaneous mechanoreceptors in the human foot. Neurosci Lett 34:289-294.

https://doi.org/10.1016/0304-3940(82)90190-2

Von Holst E, Mittelstaedt H (1950) Das reafferenzprinzip, Naturwissenschaften 37:464-476. https://doi.org/10.1007/BF00622503

Voss M, Ingram JN, Haggard P, Wolpert DM (2006) Sensorimotor attenuation by central motor command signals in the absence of movement. Nat Neurosci 9:26-7.

https://doi.org/10.1038/nn1592 
White O, Davare M, Andres M, Olivier E (2013) The role of left supplementary motor area in grip force scaling. PLoS ONE 8(12): e83812. https://doi.org/10.1371/journal.pone.0083812 Williams SR, Chapman CE (2002) Time course and magnitude of movement-related gating of tactile detection in humans. III. Effect of Motor Tasks. J Neurophysiol 88:1968-1979.

https://doi.org/10.1152/in.2002.88.4.1968

Wolpert DM, Goodbody SJ, Husain M (1998) Maintaining internal representations: the role of the human superior parietal lobe. Nat Neurosci 1:529-233. doi: 10.1038/2245.

Zatsiorsky VM, Li ZM, Latash ML (2000) Enslaving effects in multi-finger force production. Exp Brain Res 131:187-195. https://doi.org/10.1007/s002219900261 